\title{
Non-perturbative summation over 3D discrete topologies
}

\author{
Laurent Freidel* \\ Perimeter Institute for Theoretical Physics \\ 35 King street North, Waterloo N2J-2G9,Ontario, Canada \\ and \\ Laboratoire de Physique, École Normale Supérieure de Lyon \\ 46 allée d'Italie, 69364 Lyon Cedex 07, France \\ David Louapre ${ }^{\dagger}$ \\ Laboratoire de Physique, École Normale Supérieure de Lyon \\ 46 allée d'Italie, 69364 Lyon Cedex 07, France
}

(Dated: November 2, 2018)

\begin{abstract}
We construct a group field theory which realizes the sum of gravity amplitudes over all three dimensional topologies trough a perturbative expansion. We prove this theory to be uniquely Borel summable. This shows how to define a non-perturbative summation over triangulations including all topologies in the context of three dimensional discrete gravity.
\end{abstract}

*Electronic address: Ifreidel@perimeterinstitute.ca

$\dagger$ Electronic address: dlouapre@ens-lyon.fr

$\ddagger$ UMR 5672 du CNRS 


\section{INTRODUCTION}

In quantum gravity, we are interested in the computation of transition amplitudes between spatial geometries. These transition amplitudes are supposed to arise as a sum over spacetime 'geometries'. In the classical realm what is meant by geometries is clear, one usually means a smooth Lorentzian metric free of singularity on a manifold of fixed topology, possibly satisfying some asymptotic conditions. In the quantum realm we obviously have much more freedom in choosing which spacetime geometries should be included in the sum. Do we authorize the geometries to posses some singularities for instance? [1] Do we authorize topology change? One of the outstanding question in this context is wether one should or not include a sum over topology in the sum over geometries. The next outstanding question is wether this is possible in a given framework. In this paper we are going to analyze this problem in the context of discrete approach to quantum gravity, i-e dynamical triangulation and spin foam models. In dynamical triangulation one first choose an equilateral triangulation $\Delta$ of space-time with regular simplices and we assign to each triangulation a statistical weight given by the exponential of the Regge action. In spin foam models one choose a triangulation of space time and assign to it certain representation labels of the Lorentz group. This representation labels carry information on the geometry of the simplex. The transition amplitudes are constructed as a state sum, using sum over representations assigned to the geometrical building blocks of the triangulation. Historically, the first spin foam model was proposed by Ponzano and Regge [2] for 3D Euclidian quantum gravity, and their work has been recently generalized to higher dimension to give birth to the spin foam approach for quantum gravity (see [3, 4] for an overview).

$3 \mathrm{D}$ gravity is a topological theory. As a consequence, the transition amplitudes built in the Ponzano-Regge model do not depend on the choice of the triangulation. However this is no longer the case in $4 \mathrm{D}$, and one has somehow to get rid of the choice of the triangulation in order to restore the infinite number of degrees of freedom of $4 \mathrm{D}$ gravity. A proposal to do this is to sum the discretized amplitude over all possible triangulations of the manifold. A more drastic proposal is to sum over all possible triangulations regardless of the topology.

It is however commonly believed that there is no way to give sense to the sum over triangulations including all topologies. The problem appears clearly in the context of dynamical triangulations [5]. In this context the amplitude of a given 3-dimensional triangulation $\Delta$ is given by

$$
\mathcal{A}[\Delta]=\frac{1}{\operatorname{Sym}[\Delta]} \lambda^{N_{3}} \mu^{N_{1}},
$$

where $\operatorname{Sym}[\Delta]$ is a symmetry factor, and $N_{3}$ (resp. $N_{1}$ ) denote the number of tetrahedra (resp. edges) of the triangulation. The parameters $\lambda, \mu$ can be interpreted, if one uses the Regge action, in terms of the bare cosmological constant $\Lambda$ and the bare Newton constant $1 / G$

$$
\begin{array}{r}
\mu \sim \exp \left(\frac{1}{G}\right), \\
\lambda \sim \exp \left(-\Lambda-\frac{c}{G}\right),
\end{array}
$$

where $c$ is a geometrical constant. The sum over all triangulations is then

$$
Z=\sum_{N_{3}, N_{1}} \lambda^{N_{3}} \mu^{N_{1}} \mathcal{N}\left(N_{3}, N_{1}\right)
$$

with $\mathcal{N}\left(N_{3}, N_{1}\right)$ being the number of triangulations with a fixed number of tetrahedra and edges. Since $\mu \geq 1$ the partition function is bounded from below by $\sum_{N_{3}} \lambda^{N_{3}} \mathcal{N}\left(N_{3}\right)$ where $\mathcal{N}\left(N_{3}\right)$ is the total number of triangulations with a fixed number of tetrahedra. This number grows factorially with the number of tetrahedra if there is no restriction on the topology of the triangulation. Therefore a sum over triangulations does not seem to be possible in general, since this factorial growth can not be killed by any choice of $\lambda, \mu$. The solution proposed in [5] is to restrict to a fixed topology (as the 3-sphere), in order to get back to a case where the number of triangulations grows exponentially with the number of 3-simplices. So the sum over simplicial manifold can be defined in the case of a restricted topology. However, since there is no general classification of the topologies in $D>2$, we cannot expect to use this result to make sense of a sum over topologies as in 2 dimensions. One conclusion that one can draw is that one should not try to sum over topology when trying to solve quantum gravity. This is not completely satisfactory, since first, there are argument in favor of classical topology change [1], but also one would expect in a theory of quantum gravity to resolve this issue dynamically and not a priori.

There is hopefully another point of view that can be taken on this problem. It is well known since Dyson [6] that physical quantities in interacting quantum theory are non-analytic functions of the coupling constant. This translates into the fact that the perturbative expansion of any physical quantities in terms of the coupling constant 
is a divergent series. This opens the possibility of giving a meaning to the sum over triangulations if we can interpret it as a perturbative expansion of a non-perturbative quantity.

These ideas have already been used in the context of $2 \mathrm{D}$ gravity, leading to the construction of the matrix models, which realize a sum over triangulations of 2D surfaces [7]. By considering three-indices tensors instead of matrices, Ambjorn et al. [5] tried to apply these ideas to obtain a dynamical triangulation model for three-dimensional quantum gravity. Later, Boulatov and Ooguri [8, 9] have generalized these models in the form of non local field theories over group manifold. The bottom line is that a space-time triangulation can naturally be interpreted as a Feynman graph of a Group Field Theory (GFT) [10]. Moreover, the specific gravity amplitude assigned to a given triangulation is exactly given by the Feynman graph evaluation of a group field theory GFT. This was shown in the context of 3D dynamical triangulation [5], 3D Ponzano-Regge model [8] and even 4D spin foam model [11]. It is now understood that this is a general feature of spin foam models [12].

All these results lead to the conclusion that the sum over triangulations of a state sum amplitude is in fact a sum over Feynman graphs of a GFT,

$$
\sum_{\Delta} \mathcal{A}_{\text {State sum }}(\Delta)=\sum_{\Gamma} \mathcal{A}_{\mathrm{GFT}}(\Gamma) .
$$

Therefore it is not a surprise that the sum over triangulations is divergent, since it is a perturbative expansion, it should be understood as an asymptotic series. If we take for serious the fact that the sum over triangulations comes from a group field theory, this opens the possibility of giving a non perturbative meaning to the sum. This idea has been already advocated in [12] and to some extent in [5].

However it is well known that in general, the asymptotic series does not determine uniquely the resummation. One need additional non-perturbative inputs. In interacting quantum field theory, the large order behavior is determined by non-perturbative information contained in the action and in some case [13] one is able to reconstruct a nonperturbative expression from the divergent series, in a uniquely defined manner. In such cases, the divergent series is said to be uniquely Borel-summable. Our main result in this paper is to prove that such a procedure can be realized for a particular type of GFT models associated with three dimensional gravity. If the Feynman expansion of a GFT model is Borel-summable, this means it could be related to a non perturbative expression in a uniquely defined manner, giving a well-defined meaning to the equality

$$
\sum_{\Gamma} \mathcal{A}_{\mathrm{GFT}}(\Gamma)=Z_{N P}
$$

This result proves that a well-defined notion of a sum over all triangulations of all topologies is not unreasonable, as it could be achieved in particular cases.

In the context of dynamical triangulation the coupling constant $\lambda$ of the group field theory has a physical meaning, it can be interpreted as $-\exp (-\Lambda)$, where $\Lambda$ is the cosmological constant. Since it is negative, the potential is unbounded from below and it was expected that the corresponding GFT is not uniquely Borel summable in the physical regime. Therefore one can think that the theories allowing non perturbative resummation will not be physical (at least in the context of dynamical triangulations). In two dimension one can view this problem as the one preventing a non perturbative definition of string theory via matrix models.

It was therefore for us a big surprise and a relief to find out that the modification of the Ambjorn et al. model [5] we propose is uniquely Borel summable in the physical region where $\lambda>0$ (see sect. IV). In the context of three dimensional Ponzano-Regge models the value of the group field theory coupling constant model is not restricted by physical requirement and we expect the Borel summable GFT to have physical meaning without restriction.

In section II of this paper we first review the definitions of several group field theory models related to 3 dimensional gravity like the Boulatov or Ambjorn model. We present their Feynman expansions and show their relations with 3 dimensional Ponzano-Regge models or dynamical triangulations. We also discuss their convergence properties. In section III we review known facts and theorems concerning non perturbative resummation of a perturbative series. In section IV we propose a modification of the usual Ambjorn or Boulatov models in order to deal with positive potential. We show that the perturbative expansion of this model can be related to the dynamical triangulation sum with physical value of the parameters. In the last section $\mathrm{V}$ we finally prove the unique Borel summability of the model we have defined. This give us an example of an unambiguous way to sum over all triangulations hence all topologies.

\section{3D GROUP FIELD THEORY MODELS}

In this part, we review various constructions of 3D group field theory (GFT) models : the Boulatov model, leading to the Ponzano-Regge amplitude [8], a model on the non-commutative sphere, reproducing the 3D matrix model 
of Ambjorn et al.[5], and some others models with improved convergence properties, analogous to those recently introduced for 4D spin foam models $[14,15]$.

\section{A. The Boulatov model}

The Boulatov model is a field theory defined on the group manifold $S O(3)^{3}$, with some invariances imposed on the fields. The fields are asked to be real $(\bar{\Phi}=\Phi)$, symmetric

$$
\Phi\left(g_{\sigma(1)}, g_{\sigma(2)}, g_{\sigma(3)}\right)=(-1)^{|\sigma|} \Phi\left(g_{1}, g_{2}, g_{3}\right)
$$

(where $\sigma$ denotes a permutation of three elements and $|\sigma|$ its signature), and (right-) $S O(3)$ invariant

$$
\Phi\left(g_{1} g, g_{2} g, g_{3} g\right)=\Phi\left(g_{1}, g_{2}, g_{3}\right)
$$

This last requirement allows us to see the Boulatov model as a field theory on the coset $\operatorname{space~} \mathrm{SO}(3)^{3} / \mathrm{SO}(3)$. The action is non-local and is given by

$$
\begin{aligned}
S[\Phi] & =\frac{1}{2} \int_{G^{3}} d g_{1} d g_{2} d g_{3} \Phi\left(g_{1}, g_{2}, g_{3}\right)^{2} \\
& +\frac{\lambda}{4 !} \int_{G^{6}} d g_{1} d g_{2} d g_{3} d g_{4} d g_{5} d g_{6} \Phi\left(g_{1}, g_{2}, g_{3}\right) \Phi\left(g_{3}, g_{5}, g_{4}\right) \Phi\left(g_{4}, g_{2}, g_{6}\right) \Phi\left(g_{6}, g_{5}, g_{1}\right)
\end{aligned}
$$

To compute the partition function of this model in perturbative expansion, one has to express the action in terms of the Fourier coefficients of the field $\Phi$. In order to define a Fourier transform on the coset space, one first considers the transform on $\mathrm{SO}(3)^{3}$, then requires the $\mathrm{SO}(3)$-invariance. The representations of $\mathrm{SO}(3)$ are labelled by integer spin $j$, and a function on $\mathrm{SO}(3)$ can be expressed as a sum over the matrix elements of $g$ in the spin $j$ representation $D_{m n}^{j}(g)$ (see appendix A)

$$
\Phi(g)=\sum_{j, m, n} d_{j} \Phi_{j}^{m n} D_{m n}^{j}(g),
$$

where $d_{j}=2 j+1$ and $-j \leq m, n \leq j$. The Fourier coefficient is

$$
\Phi_{j}^{m n}=\int_{G} d g \Phi(g) D_{m n}^{j}\left(g^{*}\right),
$$

where $g^{*}$ denotes complex conjugation. The Fourier expansion on $\mathrm{SO}(3)^{3}$ is then

$$
\Phi\left(g_{1}, g_{2}, g_{3}\right)=\sum_{\vec{\jmath}, \vec{m}, \vec{n}} d_{\vec{\jmath}} \Phi_{\vec{\jmath}}^{\vec{m} \vec{n}} D_{\vec{m} \vec{n}}^{\vec{\jmath}}(\vec{g})
$$

where $\vec{j} \equiv\left(j_{1}, j_{2}, j_{3}\right)$ and $D_{\vec{m} \vec{n}}^{\vec{\jmath}}(\vec{g}) \equiv D_{m_{1}, n_{1}}^{j_{1}}\left(g_{1}\right) D_{m_{2}, n_{2}}^{j_{2}}\left(g_{2}\right) D_{m_{3}, n_{3}}^{j_{3}}\left(g_{3}\right)$. The $\mathrm{SO}(3)$-invariance is implemented by group averaging over the right diagonal action of $\mathrm{SO}(3)$

$$
\Phi\left(g_{1}, g_{2}, g_{3}\right)=\int d g \Phi\left(g_{1} g, g_{2} g, g_{3} g\right)
$$

Expanding the RHS using decomposition (12), then performing the integration on $g$ using the relation (A6), we obtain the Fourier decomposition of the field over $\mathrm{SO}(3)^{3} / \mathrm{SO}(3)$

$$
\Phi\left(g_{1}, g_{2}, g_{3}\right)=\sum_{\vec{\jmath}, \vec{m}, \vec{n}} A_{\vec{\jmath}}^{\vec{m}} \sqrt{d_{\vec{\jmath}}} D_{\vec{m} \vec{n}}^{\vec{\jmath}}(\vec{g}) C_{\vec{n}}^{\vec{j}},
$$

where we introduced the Fourier coefficient

$$
A_{\vec{\jmath}}^{\vec{m}}=\sum_{\vec{n}} \Phi_{\vec{\jmath}}^{\vec{m} \vec{n}} \sqrt{d_{\vec{\jmath}}} C_{\vec{n}}^{\vec{j}}
$$



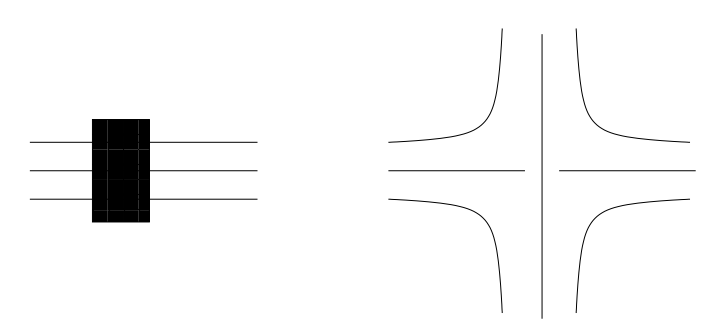

FIG. 1: Graphical representation of the propagator and potential. The black square stands for the sum over different permutations of the three lines, see (20).

and $C_{\vec{n}}^{\vec{j}}$ denotes the Wigner $3 j$-symbol (see appendix A). The symmetries asked on the fields are translated on the coefficients :

$$
\begin{aligned}
\bar{A}_{j_{1} j_{2} j_{3}}^{m_{1} m_{3}} & =(-1)^{\sum_{i}\left(j_{i}+m_{i}\right)} A_{j_{1} j_{2} j_{3}}^{-m_{1}-m_{2}-m_{3}}, \\
A_{j_{\sigma(1)} j_{\sigma(2)} j_{\sigma(3)}}^{m_{\sigma(1)} m_{\sigma(2)} m_{\sigma(3)}} & =(-1)^{|\sigma|} A_{j_{1} j_{2} j_{3}}^{m_{1} m_{2} m_{3}} .
\end{aligned}
$$

We can now compute the action (9) in terms of the Fourier coefficients. We insert the Fourier decomposition (14) in the action, and perform the integrations using (A5). The four $3 j$-symbols appearing in the potential term recombine into a $6 j$-symbol. We finally obtain the action in Fourier modes

$$
S=\frac{1}{2} \sum_{\vec{j}, \vec{n}}\left|A_{j_{1} j_{2} j_{3}}^{m_{1} m_{2} m_{3}}\right|^{2}+\frac{\lambda}{4 !} \sum_{j_{1}, \ldots j_{6}, m_{1}, \ldots m_{6}}(-1)^{\sum_{i}\left(j_{i}+m_{i}\right)} A_{j_{1} j_{2} j_{3}}^{-m_{1}-m_{2}-m_{3}} A_{j_{3} j_{5} j_{4}}^{m_{3}-m_{5} m_{4}} A_{j_{4} j_{2} j_{6}}^{-m_{4} m_{2} m_{6}} A_{j_{6} j_{5} j_{1}}^{-m_{6} m_{5} m_{1}}\left\{\begin{array}{lll}
j_{1} & j_{2} & j_{3} \\
j_{4} & j_{5} & j_{6}
\end{array}\right\} .
$$

We want to compute the partition function and correlation functions of the theory. They are formally defined in terms of the path integral

$$
Z=\int d \Phi e^{-S[\Phi]}
$$

As usual we compute this partition function as a perturbative expansion. The Feynman rules of such a group field model are very similar to those of a usual QFT. One first construct the propagator and then include the interaction perturbatively. The propagator is given by

$$
<A_{\vec{j}}^{\vec{m}} A_{\vec{j}}^{\vec{n}}>=\frac{1}{3 !} \sum_{\sigma \in \Sigma_{3}} \delta_{\vec{j}, \sigma\left(\overrightarrow{j^{\prime}}\right)} \delta_{\vec{n}, \sigma(\vec{m})}
$$

where the sum is over permutation of three elements. The full contribution can be recast into a sum over Feynman graphs, whose form is dictated by the action. For each graph we compute its Feynman amplitude by labelling the lines of the graph by representations indices; associate a weight to the lines and the vertices; then sum over representation indices. In our model the graphical form of the propagator and the vertex are given in figure 1 . The contributions of the theory at order $n$ are obtained by constructing graphs with $n$ vertices. We then label each closed line of the graph by representation indices $(j, m)$, associate to each vertex the weight given by the $6 j$-symbol, then sum over representation indices. Considering a graph with $n$ vertices and a symmetry factor $\operatorname{sym}[\Gamma]$, we obtain the amplitude

$$
\mathcal{A}[\Gamma]=\frac{(-\lambda)^{n}}{\operatorname{sym}[\Gamma]} \sum_{\{j, m\}} \prod_{v}\left\{\begin{array}{lll}
j_{1} & j_{2} & j_{3} \\
j_{4} & j_{5} & j_{6}
\end{array}\right\} .
$$

As the weight does not depend on the indices $m$ on the lines, the sum over each of the $m$ indices is trivial and equal for each line to the number of terms $\operatorname{dim} j_{l}=2 j_{l}+1$. Thus one get the amplitude

$$
\mathcal{A}[\Gamma]=\frac{(-\lambda)^{n}}{\operatorname{sym}[\Gamma]} \sum_{\{j\}} \prod_{l}\left(2 j_{l}+1\right) \prod_{v}\left\{\begin{array}{lll}
j_{1} & j_{2} & j_{3} \\
j_{4} & j_{5} & j_{6}
\end{array}\right\} .
$$

By associating a triangle to each propagator and gluing them together at vertices, one generates tetrahedra (as seen in figure 2) and in general, a Feynman graph of the theory is interpreted as a 3D triangulation. The state-sum amplitude 


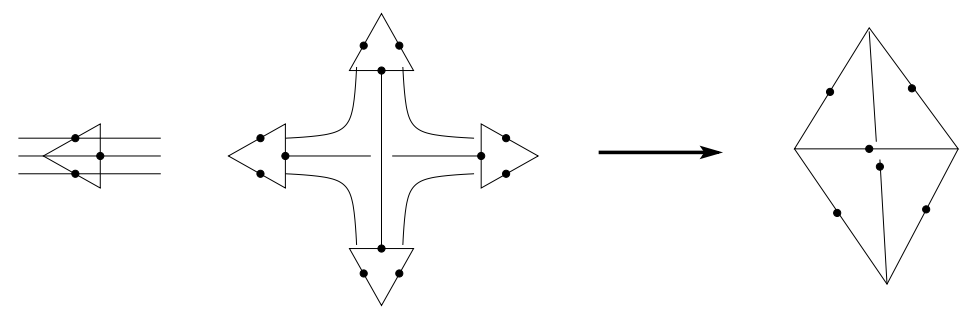

FIG. 2: Triangulation generated by the Feynman diagrams

associated to a triangulation $\Delta$ is thus

$$
\mathcal{A}[\Delta]=\frac{(-\lambda)^{n}}{\operatorname{sym}[\Delta]} \sum_{\{j\}} \prod_{e}\left(2 j_{e}+1\right) \prod_{t}\left\{\begin{array}{lll}
j_{1} & j_{2} & j_{3} \\
j_{4} & j_{5} & j_{6}
\end{array}\right\}
$$

where $e$ and $t$ label the edges and tetrahedron of the triangulation. This amplitude is exactly the Ponzano-Regge partition function associated to the triangulation $\Delta$. Moreover, all the triangulations can be obtained as Feynman graphs of the theory, thus the sum over Feynman graphs is indeed the sum over triangulations of the Ponzano-Regge amplitude.

As in standard quantum field theory, the GFT are in general faced with two kind of divergences. The first ones are the analog of the UV/IR divergences, and arise when computing the value of a given graph by summing over representation indices (momenta). The second ones are the large order divergences, arising when one try to sum all the Feynman graphs, in order to get a non-perturbative expression of a physical quantity. In this paper we will mainly concentrate on the large order divergences, and we need somehow to get rid of the UV/IR divergences. For instance, it is well known that the weight (22) is divergent for some triangulations, the divergences being associated with the vertices of the triangulation. This is a generalization of the usual fact that in standard field theory divergences are associated with the loop of the Feynman graphs (for more details on loop divergences in group field models see $[14,15]$.) As we will see at the end of this section, one way to avoid these divergences is to define spin foam models with enhanced convergence properties, in analogy with the models introduced in [14, 15]. However the Ponzano-Regge weight generated by the Boulatov model is not one of these enhanced models, and we feel that a full understanding of the notion of renormalisability for these theories should be developed. In this paper we will not dwell further on this issue and we will ignore the UV problems by working with a fix regulator and concentrate on the problem of large order divergences.

The natural regularization to choose in this context, which respects the symmetry, is to project out modes of the fields carrying a spin $j$ bigger than say $N$. So instead of the field $\Phi$ we use the projected field

$$
\Phi_{N}\left(g_{1}, g_{2}, g_{3}\right)=\sum_{\vec{\jmath} \leq N} d_{\vec{\jmath}} \sum_{\vec{m}, \vec{n}} \Phi_{\vec{\jmath}}^{\vec{m} \vec{n}} D_{\vec{m} \vec{n}}^{\vec{\jmath}}(\vec{g}) .
$$

In this case the action expressed in Fourier mode is identical to (18), except that the sum over the spins are restricted to be over $j \leq N$. One can naturally interpret this projection as arising from a group field theory defined on the non commutative sphere $S_{N}^{3}$ [16] (see [17] and the appendix B for non commutative 2-sphere $S_{N}^{2}$ ). This leads to an interpretation of the regularized group field theory model in terms of a generalized matrix models, since functions on the non commutative sphere are matrices. Another natural way to get a regularization is to define this model with the q-deformation of $\mathrm{SU}(2)$ for $q$ a root of the unity $q=e^{i \sqrt{\Lambda}}$, where the cut off $N$ in the representations is related to the cosmological constant $\Lambda$ by $2 N+1=\pi \Lambda^{-1 / 2}$.

\section{B. Group field model on the sphere and 3D matrix models}

Keeping the action (9), one can define various models by requiring different kind of invariances on the fields. For instance by considering a real symmetric field on $\mathrm{SO}(3)^{3}$ with a $\mathrm{SO}(2)^{3}$-invariance,

$$
\Phi\left(g_{1} h_{1}, g_{2} h_{2}, g_{3} h_{3}\right)=\Phi\left(g_{1}, g_{2}, g_{3}\right) \quad \forall\left(h_{1}, h_{2}, h_{3}\right) \in S O(2)^{3}
$$

one obtains a group field model on the coset space $[\mathrm{SO}(3) / \mathrm{SO}(2)]^{3}$. To implement the $\mathrm{SO}(2)^{3}$-invariance, one fixes an $\mathrm{SO}(2)$ subgroup of $\mathrm{SO}(3)$. In particular, we may consider $\mathrm{SO}(3)$ in its fundamental representation and choose a 
unit vector $v^{0}$; we then let $H$ be the $\mathrm{SO}(2)$ subgroup which leaves $v^{0}$ invariant. The space of the equivalence classes, $\mathrm{SO}(3) / \mathrm{SO}(2)$, is diffeomorphic to the 2 -sphere $S^{2}$. Thus a GFT over the group manifold $[\mathrm{SO}(3) / \mathrm{SO}(2)]^{3}$ is viewed as defined over three copies of the 2-sphere $S^{2}$. As in the case of the Boulatov model, one has to determine the Fourier transform by requiring the invariance on the Fourier expansion. If the representation is of integer spin $j$ there is a unique vector invariant under the action of the $\mathrm{SO}(2)$-subgroup, we denote it $v^{0}$. The $\mathrm{SO}(2)$-group averaging of the matrices of representation gives the projector over this invariant vector

$$
\int_{S O(2)} d h D_{m n}^{j}(h)=v_{m}^{0} v_{n}^{0}
$$

So if we define the Fourier coefficient

$$
A_{\vec{j}}^{\vec{m}}=\sqrt{d_{\vec{\jmath}}} \sum_{\vec{n}} \Phi_{\vec{j}}^{\vec{m}, \vec{n}} v_{\vec{n}}^{0}
$$

the field is expressed as

$$
\Phi\left(g_{1}, g_{2}, g_{3}\right)=\sum_{\vec{\jmath}, \vec{m}, \vec{n}} A_{\vec{m}}^{\vec{\jmath}} \sqrt{d_{\vec{\jmath}}} D_{\vec{m} \vec{n}}^{\vec{\jmath}}(\vec{g}) v_{\vec{n}}^{0} .
$$

The reality conditions are translated on the coefficients

$$
\bar{A}_{m_{1} m_{2} m_{3}}^{j_{1}, j_{2}, j_{3}}=(-1)^{\sum_{i} m_{i}} A_{-m_{1}-m_{2}-m_{3}}^{j_{1}, j_{2}, j_{3}}
$$

The action is found to be

$$
S=\frac{1}{2} \sum_{j_{1}, j_{2}, j_{3}, m_{1}, m_{2}, m_{3}}\left|A_{j_{1} j_{2} j_{3}}^{m_{1} m_{2} m_{3}}\right|^{2}+\frac{\lambda}{4 !} \sum_{j_{1} \ldots j_{6}, m_{1} \ldots m_{6}}(-1)^{\sum_{i} m_{i}} A_{j_{1} j_{2} j_{3}}^{-m_{1}-m_{2}-m_{3}} A_{j_{3} j_{5} j_{4}}^{m_{3}-m_{5} m_{4}} A_{j_{4} j_{2} j_{6}}^{-m_{4} m_{2} m_{6}} A_{j_{6} j_{5} j_{1}}^{-m_{6} m_{5} m_{1}}
$$

The Feynman rules are the same than in the previous case, and the state sum amplitude associated to a graph is given by

$$
(-\lambda)^{n} \sum_{\{j, i\}} 1=(-\lambda)^{n} \sum_{\{j\}} \prod_{e}\left(2 j_{e}+1\right)
$$

As before, this is interpreted as the amplitude associated to a 3D triangulation. This is a variant of the Ponzano-Regge model, with 1 as tetrahedron amplitude, instead of the $6 j-$ symbol.

This partition function obviously diverges and has to be regularized. As before, an elegant way to regularize this model is to consider the original model to be defined on the non-commutative sphere $S_{N}^{2}$ instead of the 2-sphere. The algebras of function on $S_{N}^{2}$ is just the space of $(N+1) \times(N+1)$ matrices. This space carries naturally a representation of $\mathrm{SU}(2)$; if we decompose it in terms of spherical harmonics it can be written as a sum over spin $j$ representation where $j \leq N$ (see appendix B). The amplitude associated to a graph becomes

$$
\begin{aligned}
\mathcal{A}[\Gamma] & =\frac{(-\lambda)^{n}}{\operatorname{sym}[\Gamma]} \sum_{\{j \leq N\}} \prod_{e}\left(2 j_{e}+1\right)=\frac{(-\lambda)^{n}}{\operatorname{sym}[\Gamma]}\left[(N+1)^{2}\right]^{(\# \text { edges })} \\
& =\frac{1}{\operatorname{sym}[\Gamma]}(-\lambda)^{(\# \text { tetrahedra })} \mu^{(\# \text { edges })}
\end{aligned}
$$

which is finite (we defined $\left.\mu \equiv(N+1)^{2}\right)$. This amplitude is the amplitude (1) obtained in 3D dynamical triangulations. However, we have to notice that to correctly interpret this GFT as defining a sum over triangulations of the 3D dynamical triangulation model, we have to set $\lambda$ negative, namely $-\lambda \sim \exp \left(-\Lambda-\frac{c}{G}\right)$, see $(3)$.

\section{Other group field theories}

The two previous group field theories were the first ones introduced in the literature [5, 8]. There has been since then a resurgence of these models when it appeared that the Barrett-Crane amplitude, which was a candidate for a 4D state sum model of quantum gravity, could be interpreted as a Feynman graph amplitude of a group field theory $[11,18]$. 
Since then, different modifications of the original 4D model have been proposed first by Perez and Rovelli [14] (note also the generalization in [19]). The purpose of these modified models was to improve the convergence properties of the Feynman graph amplitudes. We give here the 3D analogs of these models.

The general group field theory we will consider is defined on $\mathrm{SO}(3)^{3}$ for real symmetric fields, with the action

$$
\begin{aligned}
S[\Phi] & =\frac{1}{2} \int d g_{1} d g_{2} d g_{3}\left[P_{k i n} \Phi\left(g_{1}, g_{2}, g_{3}\right)\right]^{2} \\
& +\frac{\lambda}{4 !} \int d g_{1} d g_{2} d g_{3} d g_{4} d g_{5} d g_{6}\left[P_{p o t} \Phi\left(g_{1}, g_{2}, g_{3}\right)\right]\left[P_{p o t} \Phi\left(g_{3}, g_{5}, g_{4}\right)\right]\left[P_{p o t} \Phi\left(g_{4}, g_{2}, g_{6}\right)\right]\left[P_{p o t} \Phi\left(g_{6}, g_{5}, g_{1}\right)\right],
\end{aligned}
$$

where $P_{k i n}, P_{p o t}$ are operators acting on the space of fields and implementing some invariance property. For instance the Boulatov model is recovered for $P_{k i n}=P_{p o t}=P_{S O(3)}$. Where $P_{S O(3)} \Phi\left(g_{1}, g_{2}, g_{3}\right)=\int_{S O(3)} \Phi\left(g_{1} g, g_{2} g, g_{3} g\right) d g$ is the projector on the space of $S O(3)$ invariant fields. We denote by $P_{S O(2)^{3}}^{R}$ the projector on the sphere, i-e $P_{S O(2)^{3}}^{R} \Phi\left(g_{1}, g_{2}, g_{3}\right)=\int_{S O(2)^{3}} \Phi\left(g_{1} h_{1}, g_{2} h_{2}, g_{3} h_{3}\right) d h_{1} d h_{2} d h_{3}$. The Ambjorn model is recovered for $P_{k i n}=P_{p o t}=$ $P_{S O(2)^{3}}^{R}$. We can consider new group field theories that gives 3 dimensional analogs of the models introduced by Perez and Rovelli, if we use

$$
\begin{aligned}
P_{k i n} & =P_{S O(3)}^{R}, \\
P_{p o t} & =\left(P_{S O(3)}^{R} P_{S O(2)^{3}}^{R}\right)^{p} P_{k i n} .
\end{aligned}
$$

As the kinetic term is the same than in the Boulatov model, the Fourier coefficient will be the same. However, applying the projectors in $P_{\text {pot }}$ leads to the following state sum amplitude

$$
\mathcal{A}[\Gamma]=\sum_{\{j\}} \prod_{f}\left(2 j_{f}+1\right) \prod_{v}\left\{\begin{array}{lll}
j_{1} & j_{2} & j_{3} \\
j_{4} & j_{5} & j_{6}
\end{array}\right\} \Theta^{p}\left(j_{1}, j_{2}, j_{3}\right) \Theta^{p}\left(j_{3}, j_{5}, j_{4}\right) \Theta^{p}\left(j_{2}, j_{4}, j_{6}\right) \Theta^{p}\left(j_{1}, j_{5}, j_{6}\right)
$$

where $\Theta$ is given by

$$
\Theta\left(j_{1}, j_{2}, j_{3}\right)=\left[C_{\vec{n}}^{\vec{j}} v_{\vec{n}}^{0}\right]^{2}=\int d g D_{00}^{j_{1}}(g) D_{00}^{j_{2}}(g) D_{00}^{j_{3}}(g) .
$$

One can use the following majoration of $\Theta$

$$
\begin{aligned}
\Theta\left(j_{1}, j_{2}, j_{3}\right) & =C_{000}^{j_{1} j_{2} j_{3}} C_{000}^{j_{1} j_{2} j_{3}} \\
& \leq \sum_{m_{2} m_{3}} C_{0 m_{2} m_{3}}^{j_{1} j_{2} j_{3}} C_{0 m_{2} m_{3}}^{j_{1} j_{2} j_{3}}
\end{aligned}
$$

This last term in the RHS is just $1 / d_{j_{1}}$. Applying this uniformly for the three spins one gets the bound

$$
\Theta\left(j_{1}, j_{2}, j_{3}\right) \leq \frac{1}{\left(d_{j_{1}} d_{j_{2}} d_{j_{3}}\right)^{1 / 3}} .
$$

\section{NON-PERTURBATIVE RESUMMATION OF AN ASYMPTOTIC SERIES}

In this section we review well known facts on divergent series in quantum theory. We recall the physical origin of these divergences, and how to deal with them from the perturbative and non-perturbative point of view. In particular, we explain how to obtain a non-perturbative resummation of a divergent perturbative series. We illustrate these ideas on a one dimensional $\phi^{4}$ model and show the crucial role played by the instantons in this framework (see [13] for a general overview of the large order behavior in quantum field theory.)

It is known since Dyson [6] that physical quantities in interacting quantum theory are non analytic functions of the coupling constant. This is easily related to the fact that for negative values of the coupling constant, the potential fails to be bounded from below and the theory becomes unstable. The consequence is that the perturbative expansion of a physical quantity in terms of the coupling constant is a divergent series. A simple example is given by the following action

$$
S_{\lambda}(\phi)=\frac{1}{2} \phi^{2}+\frac{\lambda}{4} \phi^{4},
$$




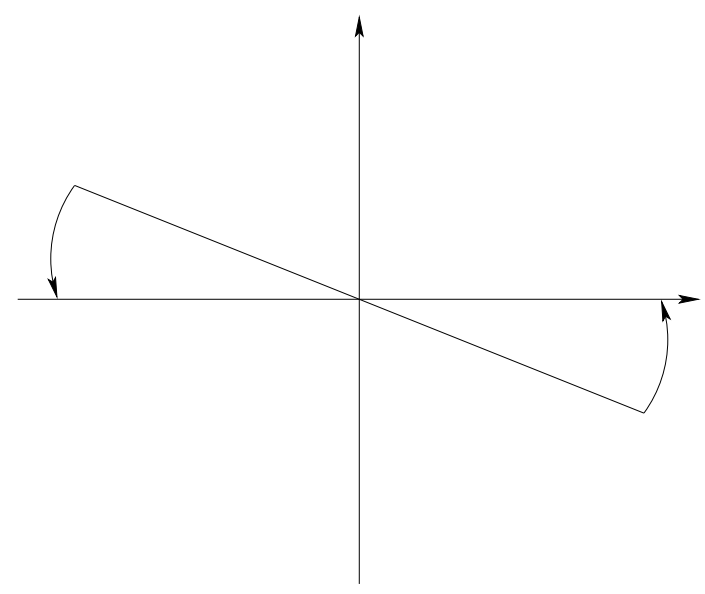

FIG. 3: Contour for the analytic continuation of $Z(\lambda)$

which is unbounded from below for negative $\lambda$. The partition function

$$
Z(\lambda)=\int_{-\infty}^{+\infty} d \phi e^{-\frac{1}{2} \phi^{2}-\frac{\lambda}{4} \phi^{4}}
$$

can be perturbatively evaluated by first expanding the integrand in terms of $\lambda$, then inverting the integration and the summation and integrating each term

$$
\int_{-\infty}^{+\infty} d \phi e^{-\frac{1}{2} \phi^{2}} \phi^{4 n}=2^{2 n+\frac{1}{2}} \Gamma\left(2 n+\frac{1}{2}\right) .
$$

The exchange of integration and summation is in fact invalid, as a result the perturbative expansion of $Z(\lambda)$

$$
Z(\lambda)=\sum_{n} \sqrt{2}(-1)^{n} \frac{\lambda^{n}}{n !} \Gamma\left(2 n+\frac{1}{2}\right)
$$

is a divergent series with the $\mathrm{n}$-th term growing like $\mathcal{C}(-1)^{n} 4^{n} n$ !.

In general, the asymptotic behavior of perturbative divergent series can be analyzed by considering classical nonperturbative objects of the theory called instantons. The instantons are complex valued solutions of the equations of motion with finite action. For instance considering the action (41), its equations of motion have two non-trivial complex solutions given by $\phi= \pm i / \sqrt{\lambda}$. The value of the action for these solutions is $S(\phi)=-\frac{1}{4 \lambda}$. These quantities are expressed using the inverse of the coupling constant, showing the non-perturbative nature of these objects. Let us see how they play a role in the large order behavior of perturbative expansions.

Consider the previous partition function (42). First, we need to show that it can be analytically continued in the complex plane, with a cut along the real negative axis. Let $\lambda=\rho e^{i \theta}$ be a complex number. We define $\tilde{Z}(\lambda)$ by

$$
\tilde{Z}(\lambda)=\int_{e^{-i \theta / 4} \mathbb{R}} d \phi e^{-\frac{1}{2} \phi^{2}-\frac{\lambda}{4} \phi^{4}}
$$

First $\tilde{Z}(\lambda)$ is well defined for all $|\theta|<\pi$, second it is an analytic continuation of $Z(\lambda)$. This is clear since for $|\theta|<\frac{\pi}{2}$, the integral along $e^{-i \theta / 4} \mathbb{R}$ is equal to the integral along $\mathbb{R}$ plus the circular contours which link these lines at infinity (see figure 3). Writing $z=R e^{i \alpha}$ on these circular contours, the integral along them is equal to

$$
\lim _{R \rightarrow \pm \infty} \int_{-\theta / 4}^{0} d \alpha e^{-\frac{1}{2} R^{2} e^{2 i \alpha}-\frac{\rho}{4} e^{i(\theta+4 \alpha)} R^{4}}
$$

which goes to zero when $R \rightarrow \pm \infty$, provided that $|\theta|<\pi / 2$. Thus the contour of integration can be shifted from $e^{-i \theta / 4} \mathbb{R}$ to $\mathbb{R}$ and the proposed expression defines the analytic continuation of $Z(\lambda)$, which can be extended to the whole complex plane, with a cut on the real negative axis. We can then define the discontinuity along this cut axis by

$$
D(-\omega)=\tilde{Z}\left(e^{i \pi} \omega\right)-\tilde{Z}\left(e^{-i \pi} \omega\right)
$$



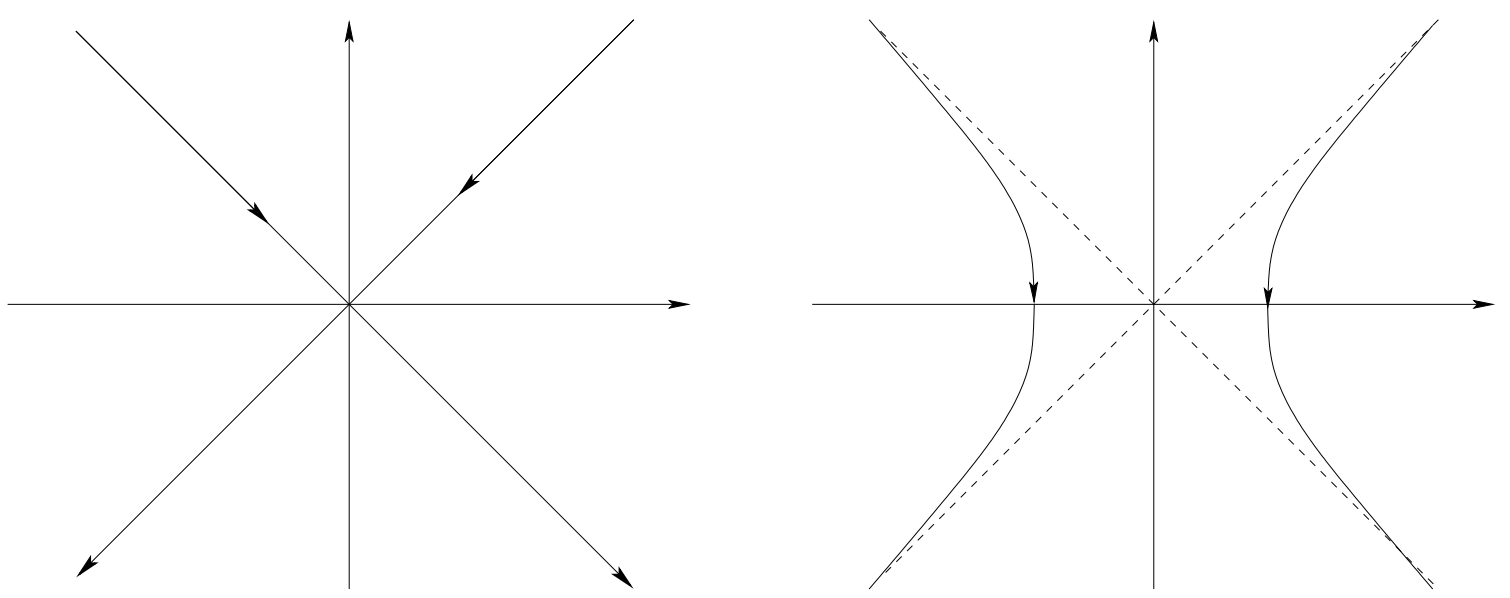

FIG. 4: a) Original contour $C$ for defining $D(-\omega)$; b) Deformed contour $C^{\prime}$ to apply the saddle point method.

for $\omega>0$. Thus $D$ is given by the integral

$$
D(-\omega)=\int_{C} d \phi e^{-\frac{1}{2} \phi^{2}+\frac{\omega}{4} \phi^{4}}
$$

where $C$ is the contour $e^{i \pi / 4} \mathbb{R}-e^{-i \pi / 4} \mathbb{R}$ pictured in figure 4 . Now $\tilde{Z}$ is related to this discontinuity by the dispersion relation

$$
\tilde{Z}(\lambda)=\frac{1}{2 i \pi} \int_{0}^{+\infty} d \omega \frac{D(-\omega)}{\omega+\lambda}
$$

We can expand this dispersion relation

$$
\tilde{Z}(\lambda)=\sum_{n} a_{n} \lambda^{n}
$$

with

$$
a_{n}=\frac{(-1)^{n}}{2 i \pi} \int_{0}^{+\infty} d \omega \frac{D(-\omega)}{\omega^{n+1}}
$$

We want to understand the large $n$ behavior of the coefficients $a_{n}$ of the series. For large $n$, the integrand in the definition of these coefficients is sharply peaked around $z=0$. This shows that the large $n$ behavior of $a_{n}$ depends on the value of $D(-\omega)$ for small $\omega$. We can change the variable of integration $\psi \equiv \sqrt{\omega} \phi$.

$$
D(-\omega)=\int_{C} d \phi e^{-\frac{1}{2} \phi^{2}+\frac{\omega}{4} \phi^{4}}=\int_{C} \frac{d \psi}{\sqrt{\omega}} e^{\frac{1}{\omega}\left(-\frac{1}{2} \psi^{2}+\frac{1}{4} \psi^{4}\right)}
$$

The value of the integral for small $\omega$ can be obtained by saddle point method. The saddle points equation is $-\psi+\psi^{3}=0$, whose non-trivial solutions are $\psi= \pm 1$. We see that these solutions corresponds to instantons solutions, since if we rescale $\psi$ by $i \sqrt{\omega}$, the saddle point equation becomes the equation of motion of the theory and the solutions becomes the instantons. The contour $C$ can be deformed into $C^{\prime}$ (see figure 4) which contains these saddle points. Expanding around these points and integrating, one gets

$$
D(-\omega) \propto e^{-1 / 4 \omega} .
$$

which is the evaluation of the action for the instantons solutions. One gets

$$
a_{n} \propto \mathcal{C}(-1)^{n} \int_{0}^{+\infty} d \omega e^{-\frac{1}{4 \omega}} \frac{1}{\omega^{n+1}} \propto(-1)^{n}\left(\frac{1}{4}\right)^{-n} n !
$$

which is in agreement with the asymptotic given for the exact computation (44). The value $(1 / 4)^{-n}$ arises from the evaluation $-1 / 4 z$ of the action on instantons solutions. This computation shows clearly how the instantons govern 
the behavior of the asymptotic series. We will now see how they are related to the way of extracting perturbative and non-perturbative information from the divergent expansions.

Even if interacting quantum theories lead to perturbative expansions which are divergent series one can use these series as approximation of the non-perturbative quantity by cutting them at an appropriate order. Consider a divergent series

$$
f(z)=\sum_{n=0}^{N-1} a_{n} z^{n}+R_{N}(z)
$$

with a rest bounded by

$$
\left|R_{N}(z)\right|<A S^{-N} N !|z|^{N}
$$

(as seen in the previous example, $S$ arise from the integration (54) and is related to the evaluation of the action for first instantons solutions.) One can use the series truncated at order $N$ as an approximation of the exact non-perturbative quantity $f(z)$, the difference between them being given by the rest $R_{N}(z)$. One can study the variations of $R_{N}(z)$ with $N$ (at fixed $z$ ) and show that the rest first decreases as $N$ increases, reaches its minimum around $N \sim S / z$, then increases and diverge to infinity. This means that the best estimation of $f(z)$ by its truncated asymptotic series is obtained at order $N \sim S / z$. One can define the accuracy of the expansion by

$$
\epsilon(z)=\operatorname{Min}_{N} R_{N}(z)
$$

Evaluating the bound (56) for $N \sim S / z$, one sees that $\epsilon(z) \sim e^{-S / z}$.

One thus know how to extract the best accurate physical information from a divergent perturbative series. However, one would like to be able to extract exact finite non-perturbative information from the divergent perturbative expansions. In order to get non-perturbative quantities from perturbative expansions, one has to reconstruct the original function $Z(\lambda)$ from an asymptotic series. However, it is easy to see that the issue of unicity of such reconstruction is not a trivial problem. For instance, if $f(z)$ as an asymptotic series $\sum a_{k} z^{k}$, then $f(z)+e^{-S / z}$ has the same one. This is not a surprise since one expects an ambiguity arising from the fact that the accuracy function is not zero, which means that the perturbative expansion does not contain all the information necessary to reconstruct the original function. Thus without additional conditions on $f$, it remains this ambiguity, there is no way to reconstruct uniquely the function from the asymptotic series. If one can find conditions on $f$ to uniquely reconstruct it from its asymptotic series, the asymptotic series is said to be uniquely Borel summable.

In that case, the unique function is recovered for instance by the Borel transform procedure. Let us consider an asymptotic series $\sum a_{k} z^{k}$. Assuming that the series

$$
B(t)=\sum \frac{a_{k}}{k !} z^{k}
$$

converges in some circle $|t|<\tau$, and that its analytic continuation admits no singularity along the positive axis, one can consider the function

$$
f(z)=\int_{0}^{+\infty} e^{-t} B(z t) d t
$$

whose perturbative expansion gives the asymptotic series $\sum a_{k} z^{k}$. The possible ambiguity in the reconstruction is manifest in the Borel transform procedure in the fact that the integral (59) can not be unambiguously performed if the Borel transform $B(t)$ admits poles along the positive axis. In this case we will have to specify how we go around the poles and different prescription will differ by terms of the type $e^{-S / z}$.

The Sokal-Nevalinna's improvement of Watson theorem [20] gives specific criteria on $f$ in order for its asymptotic series to be uniquely Borel summable.

Theorem 1 (Sokal-Nevalinna) Let $f$ be analytic in the circle $C_{R}=\left\{z \mid \Re\left(z^{-1}\right)>R^{-1}\right\}$ (see figure 5) and having an asymptotic expansion

$$
f(z)=\sum_{k=0}^{N-1} a_{k} z^{k}+R_{N}(z)
$$

with

$$
\left|R_{N}(z)\right| \leq A S^{-N} \Gamma(N+b)|z|^{N}
$$

then the asymptotic series is uniquely Borel summable and $f(z)$ can be uniquely reconstructed from it. 

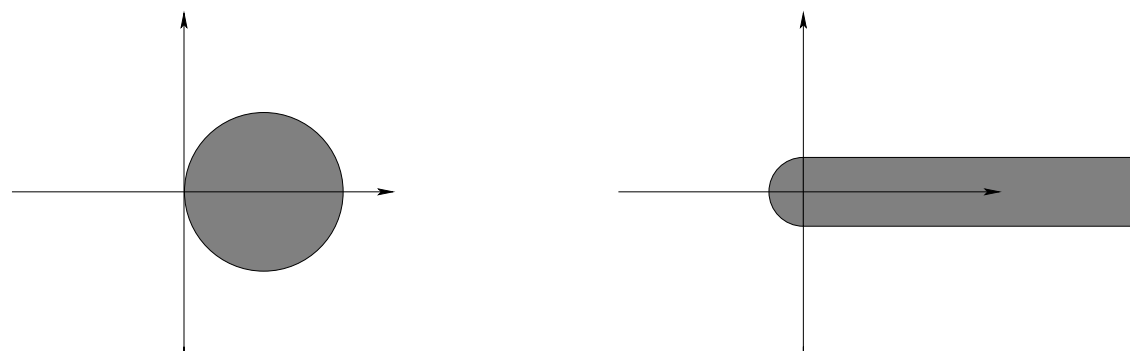

FIG. 5: Domain $C_{R}$ of analyticity for $f$, and the corresponding domain of analyticity for its Borel transform $B_{f}$, which contains an open neighbourhood of the real positive axis.

The proof of the theorem can be outlined as follows. Suppose we have two functions $f_{1}$ and $f_{2}$ having the desired asymptotic expansion and satisfying the hypothesis of the theorem. The difference between these two functions is given by their rests and one can bound these difference using (61). Our aim is to prove that this difference is null. Using the minimum on $N$, it can be bounded by the accuracy of the asymptotic expansion

$$
\epsilon(z) \sim e^{-S /|z|} .
$$

Any such function analytic in the region $C_{R}$ is in fact null. For instance the function $e^{-S / z}$ is not analytic in $C_{R}$ : let us consider the path $z(t)=t e^{i(\pi / 2-t)}$ (which is in $C_{R}$ for $t<R$ ). This path approaches $z=0$ as $t=0$ where as $\mathfrak{R}(1 / z(t))=2 \sin t / t \rightarrow 2$ so $\left|\exp ^{-S / z(t)}\right|$ doesn't approach 0 . From the point of view of the reconstruction via the Borel transform, the analyticity in $C_{R}$ ensures the analyticity of the Borel transform on a strip like region around the real positive axis (see figure 5). Thus there is no ambiguity in the choice of the contour along the real positive axis.

Coming back to our $\Phi^{4}$ example, with these criteria one can prove that the partition function (42) has an asymptotic expansion which is Borel summable. In fact we have already prove the criteria in our study of the behavior of the perturbative expansion : we have an analytic continuation in the cut complex plane which proves the first criterion and an asymptotic expression of the coefficients of the perturbative expansion which gives the desired bound for the second criterion. However, we will give now independent proofs of these criteria which are simpler and can be adapted to the GFT case.

Concerning the analyticity in the domain $C_{R}$, if consider $\lambda \in \mathbb{C}$, in the region $\Re(\lambda)>0$, we have

$$
\left|e^{-\frac{\lambda}{4} \phi^{4}}\right|=e^{-\frac{\Re(\lambda)}{4} \phi^{4}}<1 .
$$

Thus we get the bound

$$
\left|\int e^{-\frac{1}{2} \phi^{2}-\frac{\lambda}{4} \phi^{4}}\right|<\int e^{-\frac{1}{2} \phi^{2}},
$$

which ensures the convergence of the integral for $\mathfrak{R}(\lambda)>0$. In particular, $Z(\lambda)$ is analytic in a region like the $C_{R}$ needed in the first criterion. Concerning the second criterion, we start from this usual expression for the Taylor rest

$$
R_{N}(\lambda)=\frac{\lambda^{N}}{(N-1) !} \int_{0}^{1} d t(1-t)^{N-1} \frac{d^{N} Z}{d \lambda^{N}}(\lambda t) .
$$

We use the same bound (64) for $\lambda \in C_{R}$ to obtain

$$
\begin{aligned}
\left|\frac{d^{N} Z}{d \lambda^{N}}(\lambda t)\right| & =\left|\int_{-\infty}^{+\infty} d \phi e^{-\frac{1}{2} \phi^{2}-\frac{\lambda t}{4} \phi^{4}} \frac{\phi^{4 N}}{4^{N}}\right| \\
& \leq \int_{-\infty}^{+\infty} d \phi e^{-\frac{1}{2} \phi^{2}} \frac{\phi^{4 N}}{4^{N}} \\
& \leq \sqrt{2} \Gamma\left(2 N+\frac{1}{2}\right)
\end{aligned}
$$

This gives for the Taylor rest the bound

$$
\left|R_{N}(\lambda)\right| \leq \frac{\lambda^{N}}{N !} \sqrt{2} \Gamma\left(2 N+\frac{1}{2}\right)
$$


Due to the multiplicative relation on the $\Gamma$-function $\Gamma(2 x)=\frac{1}{\sqrt{2 \pi}} 2^{2 x-1 / 2} \Gamma(x) \Gamma(x+1 / 2)$, one can find a bound

$$
\frac{\Gamma\left(2 N+\frac{1}{2}\right)}{N !}<C A^{N} \Gamma(N+b)
$$

which leads a bound of the required form for the rest. The partition function satisfies the two Sokal criteria, hence its perturbative expansion is uniquely Borel summable.

\section{MODIFICATION OF THE GROUP FIELD THEORY MODEL}

In this part we present a new type of group field models, related to the previous ones, and obtained by changing the potential term in the action. To determine this potential term, we are guided by the following ideas. First of all, the previous study of a simple quantum mechanical case has shown the crucial role played by the positivity of the potential in the proofs of Borel-summability. The tetrahedral potential of action (9) has to be modified since it is not positive. Moreover, it is natural from renormalisation group arguments to include all the potential terms of identical degree, which are allowed by the symmetries of the model (see appendix $\mathrm{C}$ for a comment on the divergent graphs which a priori need to be renormalized). From the point of view of the Feynman graph expansion, this is mirrored in the fact that the tetrahedron (which appears in the interpretation of the Feynman graphs as triangulations) is not the only natural three dimensional object constructible from 4 triangles. One can construct another object, the "pillow" (see figure 6).
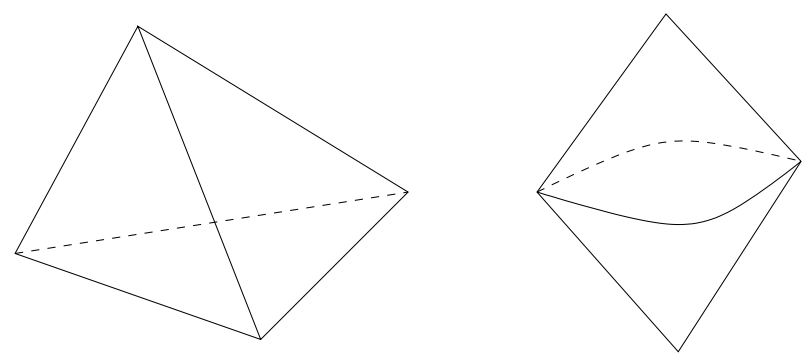

FIG. 6: The tetrahedron and the pillow : the two objects constructible from 4 triangles

Our strategy is thus to include the corresponding interaction term in the GFT. We consider a general interaction term

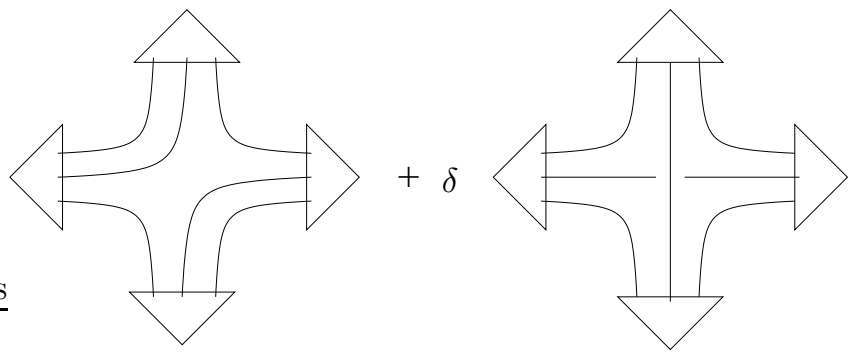

which corresponds to a total action

$$
\begin{aligned}
S[\Phi]=\frac{1}{2} \int_{G^{3}} & \Phi\left(g_{1}, g_{2}, g_{3}\right)^{2} \\
+\frac{\lambda}{4 !} \int_{G^{6}} & {\left[\Phi\left(g_{1}, g_{2}, g_{3}\right) \Phi\left(g_{3}, g_{5}, g_{4}\right) \Phi\left(g_{4}, g_{5}, g_{6}\right) \Phi\left(g_{6}, g_{2}, g_{1}\right)\right.} \\
& \left.+\quad \delta \Phi\left(g_{1}, g_{2}, g_{3}\right) \Phi\left(g_{3}, g_{5}, g_{4}\right) \Phi\left(g_{4}, g_{2}, g_{6}\right) \Phi\left(g_{6}, g_{5}, g_{1}\right)\right]
\end{aligned}
$$

Recall now that we are looking for a positive potential. We will determine the values of $\delta$ giving a positive potential. The strategy is to rewrite the total potential as a scalar product involving a linear operator whose eigenvalues are 


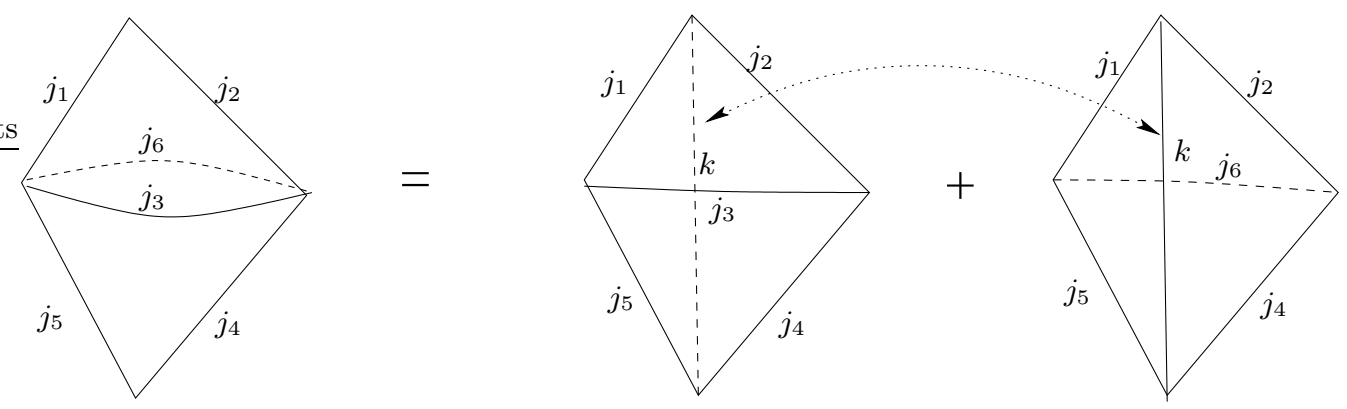

FIG. 7: A pillow is obtained by the gluing of two tetrahedra along two common faces, introducing an additional internal edge $k$

under control. Let us define the object

$$
A\left(g_{1}, g_{2}, g_{5}, g_{4}\right)=\int_{G} d g \Phi\left(g_{1}, g_{2}, g\right) \Phi\left(g, g_{5}, g_{4}\right)
$$

The pillow term is found to be

$$
\int_{G^{4}} A\left(g_{1}, g_{2}, g_{5}, g_{4}\right) A\left(g_{1}, g_{2}, g_{5}, g_{4}\right)=\langle A, A\rangle
$$

while the tetrahedron term can be written as

$$
\int_{G^{4}} A\left(g_{1}, g_{2}, g_{5}, g_{4}\right)[\mathcal{T} A]\left(g_{4}, g_{2}, g_{5}, g_{1}\right)=\langle A, \mathcal{T} A\rangle
$$

where $\mathcal{T}$ is the just the linear operator acting on $A$

$$
\mathcal{T}: A\left(g_{1}, g_{2}, g_{5}, g_{4}\right) \rightarrow A\left(g_{4}, g_{2}, g_{5}, g_{1}\right) .
$$

This operator $\mathcal{T}$ is a permutation, thus $\mathcal{T}^{2}=I d$ and its eigenvalues are simply \pm 1 . The total interaction term we defined is thus

$$
\langle A,[I d+\delta \mathcal{T}] A\rangle
$$

whose eigenvalues are $1 \pm \delta$. We obtained a positive potential for $|\delta| \leq 1$. This complete the construction of the modified potential term.

At first sight, one could say that such a modification of the potential term will lead to a drastic modification of the models, first because we will sum over different kind of triangulations, second because the weights will be different. We will see that the modification is not so drastic. We can first observe that geometrically, a pillow is no more than the gluing of two tetrahedra, along two common faces (see figure 7). This allows us to relate in unique manner each 'generalized' triangulation (made of pillows and tetrahedra) to a 'real' triangulation (made only of tetrahedra) by replacing each pillow by a pair of tetrahedra glued along two common faces. The total sum generated by the perturbative expansion can be reorganized by putting together the weights of all the generalized triangulations related to a given real triangulation, and summing only over the real triangulations. The real triangulations containing pairs of tetrahedra glued along two common faces are called 'irregular', while the other ones are called 'regular'. We can see that this reorganization concerns only the irregular triangulations.

Our results are the following. We consider the modifications of the Ambjorn and Boulatov GFT given by the action (71). In both cases the perturbative expansion of the partition function is uniquely Borel summable. In the case of the modified Ambjorn model, the perturbative expansion is written

$$
Z(\lambda, \mu)=\sum_{\Delta \in T_{\text {reg }}} \frac{1}{\operatorname{Sym}[\Delta]} \lambda^{N_{3}} \mu^{N_{1}}+\sum_{\Delta \in T_{\text {irreg }}} \frac{1}{\operatorname{Sym}[\Delta]} \lambda^{N_{3}-k} \mu^{N_{1}-k}\left(\mu \lambda-\frac{1}{2}\right)^{k},
$$


where $\mu \equiv(N+1)^{2}, N$ being the cut-off. The first sum is over regular triangulations, while the second is over irregular ones. $N_{3}$ (resp. $N_{1}$ ) is the number of tetrahedra (resp. edges) in $\Delta, k$ denotes the number of pairs of tetrahedra glued along two common faces in the irregular triangulations. In the modified Boulatov model, the perturbative expansion can be written

$$
Z(\lambda)=\sum_{\Delta \in T_{\text {reg }}} \frac{\lambda^{n}}{\operatorname{Sym}[\Delta]} Z_{P R}[\Delta]+\sum_{\Delta \in T_{\text {irreg }}} \frac{\lambda^{n-k}(\lambda-1 / 2)^{k}}{\operatorname{Sym}[\Delta]} Z_{P R}[\Delta] .
$$

where $n$ is the number of tetrahedra in the triangulation $\Delta, k$ is the number of pairs of tetrahedra glued along two common faces in an irregular triangulation and $Z_{P R}[\Delta]$ is the regularized Ponzano-Regge amplitude, for the triangulation $\Delta$. These two formulas have been written for the parameter $\delta=-1$ in the potential (see 71), but we will give a general expression valid for $|\delta|<1$ in the proof in the next section.

Several remarks can be made. First, despite the modification made on the GFT models, we still obtain a perturbative expansion which can be written as a sum over triangulations. Moreover, for all regular triangulations which do not contain any pair of tetrahedra glued along two common faces, these weights are the same than the original models. The weight are modified for the irregular triangulations and can even be cancelled by an appropriate choice of the coupling constant, namely $\lambda=1 / 2$ in the Boulatov model or $\mu \lambda=1 / 2$ in the dynamical triangulation model. In addition, our modification lead to another surprising consequence. The modification of the potential we choose leads to the fact that the factor depending on the coupling constant is now $\lambda^{n}$ instead of $(-\lambda)^{n}$, as it was the case for the originals models. Thus the relevant regime is for positive values of $\lambda$, namely the physical region of convergence, where the GFT is well-defined. Our choice of positive potential allows us not only to prove the Borel summability of the model, but also to have a well-defined GFT for the values of the coupling constant which are physically relevant.

\section{PROOF OF THE BOREL-SUMMABILITY OF THE PERTURBATIVE EXPANSION}

In this part we prove the main result, namely the unique Borel summability of the two modified models, and the fact that the resulting perturbative expansions are rewritten as slight modifications of the original models. The proofs of Borel summability are closed to those given in the simple quantum mechanical $\Phi^{4}$ case at the end of section III.

\section{A. Sokal criteria for the modified models}

The analyticity of the partition function for $\mathfrak{R}(\lambda) \geq 0$ follows directly from the positivity of the potential, using the same bound than in the $\Phi^{4}$ case, eq.(64). This proves the first criterion.

We consider now the Fourier expansion of these models. For the 3D matrix model, we find

$$
\begin{aligned}
& S=\frac{1}{2} \sum_{\vec{\jmath}, \vec{m}}\left|A_{\vec{\jmath}}^{\vec{m}}\right|^{2}+\frac{\lambda}{4 !} \sum_{j_{1} \ldots j_{6}, m_{1} \ldots m_{6}}(-1)^{\sum_{i} m_{i}}\left(A_{j_{1} j_{2} j_{3}}^{-m_{1}-m_{2}-m_{3}} A_{j_{3} j_{5} j_{4}}^{m_{3}-m_{5} m_{4}} A_{j_{4} j_{5} j_{6}}^{-m_{4} m_{5} m_{6}} A_{j_{6} j_{2} j_{1}}^{-m_{6} m_{2} m_{1}}\right. \\
& \left.+\delta \quad A_{j_{1} j_{2} j_{3}}^{-m_{1}-m_{2}-m_{3}} A_{j_{3} j_{5} j_{4}}^{m_{3}-m_{5} m_{4}} A_{j_{4} j_{2} j_{6}}^{-m_{4} m_{2} m_{6}} A_{j_{6} j_{5} j_{1}}^{-m_{6} m_{5} m_{1}}\right)
\end{aligned}
$$

For the Boulatov model, we find

$$
\begin{aligned}
& S=\frac{1}{2} \sum_{\vec{j}, \vec{n}}\left|A_{\vec{\jmath}}^{\vec{m}}\right|^{2}+\frac{\lambda}{4 !} \sum_{j_{1}, \ldots j_{6}, m_{1}, \ldots m_{6}}(-1)^{\sum_{i}\left(j_{i}+m_{i}\right)} \\
& \left(A_{j_{1} j_{2} j_{3}}^{-m_{1}-m_{2}-m_{3}} A_{j_{3} j_{5} j_{4}}^{m_{3}-m_{5} m_{4}} A_{j_{4} j_{5} j_{6}}^{-m_{4} m_{5} m_{6}} A_{j_{6} j_{2} j_{1}}^{-m_{6} m_{2} m_{1}} \theta_{j_{1} j_{2} j_{3}} \theta_{j_{1} j_{2} j_{6}} \theta_{j_{4} j_{5} j_{3}} \theta_{j_{4} j_{5} j_{6}} \frac{\delta_{j_{3}, j_{6}}}{2 j_{3}+1}\right. \\
& \left.+\delta \quad A_{j_{1} j_{2} j_{3}}^{-m_{1}-m_{2}-m_{3}} A_{j_{3} j_{5} j_{4}}^{m_{3}-m_{5} m_{4}} A_{j_{4} j_{2} j_{6}}^{-m_{4} m_{2} m_{6}} A_{j_{6} j_{5} j_{1}}^{-m_{6} m_{5} m_{1}}\left\{\begin{array}{lll}
j_{1} & j_{2} & j_{3} \\
j_{4} & j_{5} & j_{6}
\end{array}\right\}\right)
\end{aligned}
$$

where $\theta_{a b c}=1$ if $a, b, c$ satisfies the triangular inequalities, 0 if not.

In order to clarify the notations and give a uniform proof for the two cases, we separate the coefficients into real and imaginary parts. All the real coefficients we obtained are not independent due to the relations $(16,17)$. We denote by $\left\{A_{I}, I=1 \ldots d\right\}$ a set of independent coefficients ( $d$ is related to the cutoff $N$ and behaves roughly as $N^{6}$, as it stands for the six indices $\left(j_{1}, j_{2}, j_{3}, m_{1}, m_{2}, m_{3}\right)$, up to symmetry relations). The action expressed in Fourier modes can be 
rewritten as

$$
S=\frac{1}{2} \sum_{I} A_{I}^{2}+\frac{\lambda}{4 !} \sum_{I J K L} A_{I} A_{J} M^{I J K L} A_{K} A_{L}
$$

with $M^{I J K L}$ a four indices matrix representing the potential, whose positivity is translated into

$$
\sum_{I J K L} A_{I} A_{J} M^{I J K L} A_{K} A_{L} \geq 0 \forall A_{I}
$$

The partition function is

$$
Z(\lambda)=\int_{\mathbb{R}^{d}}\left[\prod_{I} d A_{I}\right] \exp \left\{-\frac{1}{2} \sum_{I} A_{I}^{2}-\frac{\lambda}{4 !} \sum_{I J K L} A_{i} A_{J} M^{I J K L} A_{K} A_{L}\right\}
$$

We want to consider the asymptotic expansion of $Z(\lambda)$

$$
Z(\lambda)=\sum_{p=0}^{n-1} a_{p} \lambda^{p}+R_{n}(\lambda)
$$

We use the expression (65) for the Taylor rest. Consider the expression for $\frac{d^{n} Z}{d \lambda^{n}}$ obtained by deriving $n$ times the original expression (83)

$$
\frac{d^{n} Z}{d \lambda^{n}}(\lambda t)=\int_{\mathbb{R}^{d}}\left[\prod_{I} d A_{I}\right] \exp \left[-\frac{1}{2} \sum_{I} A_{I}^{2}-\frac{\lambda t}{4 !} \sum_{I J K L} A_{I} A_{J} M^{I J K L} A_{K} A_{L}\right]\left(-\frac{1}{4 !} \sum_{I J K L} A_{I} A_{J} M^{I J K L} A_{K} A_{L}\right)^{n}
$$

One look for a bound of the module of the integrand. The real part of the potential is positive for $\lambda \in C_{R}$, thus

$$
\left|\exp \left[-\frac{1}{2} \sum_{I} A_{I}^{2}-\frac{\lambda t}{4 !} \sum_{I J K L} A_{I} A_{J} M^{I J K L} A_{K} A_{L}\right]\right|<\exp \left[-\frac{1}{2} \sum_{I} A_{I}^{2}\right]
$$

Thus one obtains the following bound

$$
\left|\frac{d^{n} Z}{d \lambda^{n}}(\lambda t)\right| \leq \int_{\mathbb{R}^{d}}\left[\prod_{I} d A_{I}\right] \exp \left[-\frac{1}{2} \sum_{I} A_{I}^{2}\right]\left|\frac{1}{4 !} \sum_{I J K L} A_{I} A_{J} M^{I J K L} A_{K} A_{L}\right|^{n}
$$

Let us denote

$$
S=\operatorname{Sup}_{I J K L}\left|M^{I J K L}\right|
$$

One gets the bound

$$
\left|\frac{d^{n} Z}{d \lambda^{n}}(\lambda t)\right| \leq \frac{S^{n}}{(4 !)^{n}} \int_{\mathbb{R}^{d}}\left[\prod_{I} d A_{I}\right] \exp \left[-\frac{1}{2} \sum_{I} A_{I}^{2}\right]\left(\sum_{I J K L}\left|A_{I} A_{J} A_{K} A_{L}\right|\right)^{n}
$$

The RHS integral is symmetric over the $A_{I}$, thus one can write it as $d$ ! times the integral over the domain $A_{1}>A_{2}>$ $\ldots>A_{d}$ (recall that $d$ denotes the total number of Fourier modes and depends polynomially on the cut-off $N$ ). In that case,

$$
\sum_{I J K L}\left|A_{I} A_{J} A_{K} A_{L}\right|<d^{4}\left|A_{1}\right|^{4}
$$

Then we can decouple the integrals over the $A_{I}$ on LHS of (89) and extend the integral for $I \neq 1$ in order to get $d-1$ gaussian integrals. The integral over $A_{1}$ gives a $\Gamma$-function

$$
\left|\frac{d^{n} Z}{d \lambda^{n}}(\lambda t)\right| \leq d !\left(\frac{S d^{4}}{4 !}\right)^{n}\left(\int_{\mathbb{R}} d A_{1} e^{-\frac{1}{2} A_{1}^{2}} A_{1}^{4 n}\right)\left(\int_{\mathbb{R}} d A e^{-\frac{1}{2} A^{2}}\right)^{d-1}
$$


and

$$
\left|\frac{d^{n} Z}{d \lambda^{n}}(\lambda t)\right| \leq\left(\sqrt{2} d ! \pi^{\frac{d-1}{2}}\right)\left(\frac{S d^{4}}{3 !}\right)^{n} \Gamma\left(2 n+\frac{1}{2}\right)
$$

Coming back to the expression of the Taylor rest (65), one gets a bound

$$
\left|R_{n}(\lambda)\right| \leq \mathcal{C}\left(\frac{S d^{4}}{3 !}\right)^{n} \frac{\Gamma\left(2 n+\frac{1}{2}\right)}{n !}|\lambda|^{n}
$$

which is of the required form, with constants depending on $d$, hence on the cut-off $N$. The perturbative expansion of our model satisfies the two Sokal criteria and thus is uniquely Borel summable.

\section{B. Reorganization of the perturbative expansion}

We now analyze the consequences of the introduction of the pillow interaction term on the expression of the sum over triangulations. The perturbative expansions of modified GFT models give us a sum over triangulations made with pillows and tetrahedra. However, we have seen that geometrically a pillow is obtained by gluing together two tetrahedra along two common faces.

In the matrix model case, the amplitude associated to a pillow is

$$
A_{\text {pillow }}=-\lambda
$$

while the amplitude for two tetrahedra glued along two common faces (see figure 7) is

$$
A_{2 \text { tetra }}=(-\delta \lambda)^{2} \sum_{k \leq N}(2 k+1)=(\delta \lambda)^{2}(N+1)^{2}
$$

Thus, if we compare the total amplitude for a (generalized) triangulation $\tilde{\Delta}$ containing $k$ pillows to the related real triangulation $\Delta$ obtained by replacing all the pillows by pairs of tetrahedra, we obtain (recall $\left.\mu=(N+1)^{2}\right)$

$$
Z[\tilde{\Delta}]=\left(-\frac{1}{2} \frac{1}{\lambda \delta^{2} \mu}\right)^{k} Z[\Delta]
$$

The one-half factor comes from the fact that the symmetry factor for two tetrahedra is twice the symmetry factor for one pillow. Consider a real triangulation $\Delta$ made only with $N_{3}$ tetrahedra and $N_{1}$ edges, and such that $2 k$ of its tetrahedra are glued by pair along two of their common faces. There are several generalized triangulations related to it : $C_{k}^{1}$ (binomial coefficients) triangulations with one pair of such tetrahedra replaced by a pillow, $C_{k}^{2}$ triangulations with two such pairs replaced by pillow etc...Counting together all their weights we obtain

$$
Z[\Delta]=(-\delta \lambda)^{N_{3}}\left(1+\left(-2 \delta^{2} \lambda \mu\right)^{-1} C_{k}^{1}+\left(-2 \delta^{2} \lambda \mu\right)^{-2} C_{k}^{2}+\ldots+\left(-2 \delta^{2} \lambda \mu\right)^{-k} C_{k}^{k}\right) \mu^{N_{1}}=(-\delta \lambda)^{N_{3}}\left(1-\left(2 \delta^{2} \lambda \mu\right)^{-1}\right)^{k} \mu^{N_{1}}
$$

This shows that one can reorganize our sum as a sum over real triangulations of the amplitude $(-\delta \lambda)^{N_{3}} \mu^{N_{1}}$, except the fact that an irregular triangulation containing $k$ pairs of tetrahedra glued together along two common faces has a weight modified by a factor $\left(1-\left(2 \delta^{2} \lambda \mu\right)^{-1}\right)^{k}$. Which gives

$$
Z(\lambda, \mu)=\sum_{\Delta} \frac{1}{\operatorname{Sym}[\Delta]}(-\delta \lambda)^{N_{3}-k} \mu^{N_{1}-k}\left(-\delta \mu \lambda+\frac{1}{2 \delta}\right)^{k} .
$$

Recall that the positivity of the potential is valid for $|\delta| \leq 1$. For instance for $\delta=-1$, we recover the result (77) announced in section IV.

The same reorganization can be performed on the perturbative expansion of the modified Boulatov model. The amplitude associated to a pillow is

$$
A_{\text {pillow }}=-\lambda \frac{\delta_{j_{3}, j_{6}}}{2 j_{3}+1}
$$


The amplitude associated to the gluing of two tetrahedra along two common faces (see figure 7) is

$$
A_{2 \text { tetra }}=(-\delta \lambda)^{2} \sum_{k}\left(2 j_{k}+1\right)\left\{\begin{array}{lll}
j_{1} & j_{2} & k \\
j_{4} & j_{5} & j_{3}
\end{array}\right\}\left\{\begin{array}{lll}
j_{1} & j_{2} & k \\
j_{4} & j_{5} & j_{6}
\end{array}\right\}=(\delta \lambda)^{2} \frac{\delta_{j_{3}, j_{6}}}{2 j_{3}+1}
$$

due to the orthogonality relation. As before, this means that a triangulation containing a pillow has an amplitude which is $\left(1-2 \delta^{2} \lambda\right)^{-1}$ times the amplitude of the same triangulation, with the pillow replaced by two tetrahedra glued along two common faces. We reorganize the sum in the same way than before and obtain

$$
Z(\lambda)=\sum_{\Delta} \frac{1}{\operatorname{Sym}[\Delta]}(-\delta \lambda)^{n}\left(1-\frac{1}{2 \delta^{2} \lambda}\right)^{k} Z_{P R}[\Delta]=\sum_{\Delta} \frac{1}{\operatorname{Sym}[\Delta]}(-\delta \lambda)^{n-k}\left(-\delta \lambda+\frac{1}{2 \delta}\right)^{k} Z_{P R}[\Delta] .
$$

The result (78) given in section IV is recovered for $\delta=-1$.

\section{CONCLUSION}

In this paper we have studied the possibility of defining the sum over topologies of three dimensional gravity in the context of dynamical triangulation or spin foam models. We have provided an example of a group field theory model which is uniquely Borel summable in the physical regime of the theory. In order to do so we have used the fact that three dimensional triangulations are generated by a group field theory with a quartic potential and we have shown that this potential can be naturally modified in order to be positive. Therefore, the message we wanted to convey here is that it is possible to resum the sum over triangulation if one accepts to take for serious the non perturbative information provided to us by the GFT, namely the instantonic solutions. One can still question what is the physical content of this non perturbative information. This is a direction of investigation we are following. Finally, 4 dimensional triangulations are generated by quintic potentials and we may think that there is no natural modification of this potential leading to a uniquely resummable theory. This is an issue that deserves to be studied and could mean that we need more non-perturbative input in this case in order to give a meaning to the resummed series.

Acknowledgments : We would like to thank J. Ambjorn for a discussion. D. L. is supported by a MENRT grant and Eurodoc program from Région Rhône-Alpes. L. F. is supported by CNRS and an ACI-Blanche grant.

\section{APPENDIX A: REPRESENTATIONS OF SO(3) AND RACAH-WIGNER SYMBOLS}

The irreducible unitary inequivalent representations of $\mathrm{SU}(2)$ are labelled by a half-integer $j$. The representations of $\mathrm{SO}(3)$ are just the integer spin representation of $\mathrm{SU}(2)$. The space of representation is denoted $V_{j}$ and has dimension $\operatorname{dim}_{j}=2 j+1$. All the representations are self-dual $V_{j}^{*}=V_{j}$. The matrix of representations are denoted $D_{m n}^{j}(g)$. We have the relation

$$
\bar{D}_{m n}^{j}(g)=(-1)^{m-n} D_{-m-n}^{j}(g)
$$

Given three representations $V_{j_{1}}, V_{j_{2}}, V_{j_{3}}$, it exists only one (up to normalization) intertwiner $\imath: V_{j_{1}} \otimes V_{j_{2}} \otimes V_{j_{3}} \rightarrow \mathbb{C}$. One can express it by using the Wigner $3 j$-symbol whose normalization is

$$
\sum_{\vec{m}} C_{\vec{m}}^{\vec{j}} C_{\vec{m}}^{\vec{j}}=1
$$

The Racah-Wigner $6 j$ symbol is defined by

$$
\left\{\begin{array}{lll}
j_{1} & j_{2} & j_{3} \\
j_{4} & j_{5} & j_{6}
\end{array}\right\}=\sum_{m_{i}}(-1)^{j_{4}+j_{5}+j_{6}+m_{4}+m_{5}+m_{6}} C_{m_{1} m_{2} m_{3}}^{j_{1} j_{2} j_{3}} C_{m_{5}-m_{6} m_{1}}^{j_{5} j_{6} j_{1}} C_{m_{6}-m_{4} m_{2}}^{j_{6} j_{4} j_{2}} C_{m_{4}-m_{5} m_{3}}^{j_{4} j_{5} j_{3}}
$$

It satisfies the orthogonality relation

$$
\sum_{j_{6}}\left(2 j_{3}+1\right)\left(2 j_{6}+1\right)\left\{\begin{array}{lll}
j_{1} & j_{2} & j_{3} \\
j_{4} & j_{5} & j_{6}
\end{array}\right\}\left\{\begin{array}{lll}
j_{1} & j_{2} & j_{3}^{\prime} \\
j_{4} & j_{5} & j_{6}
\end{array}\right\}=\delta_{j_{3} j_{3}^{\prime}} \theta_{j_{1} j_{2} j_{3}} \theta_{j_{3} j_{4} j_{5}}
$$


where $\theta_{a b c}=1$ if $a, b, c$ satisfy the triangular inequalities, 0 in the contrary case. The fact that the integral of a matrix representation gives a projection on the space of invariant vectors leads to the following relations (using the normalized Haar measure)

$$
\int_{\mathrm{SU}(2)} d g D_{m n}^{j}(g) \bar{D}_{m^{\prime} n^{\prime}}^{j^{\prime}}(g)=\frac{1}{d_{j}} \delta^{j j^{\prime}} \delta_{m m^{\prime}} \delta_{n n^{\prime}}
$$

and

$$
\int d g D_{m_{1} n_{1}}^{j_{1}}(g) D_{m_{2} n_{2}}^{j_{2}}(g) D_{m_{3} n_{3}}^{j_{3}}(g)=C_{\vec{m}}^{\vec{j}} C_{\vec{n}}^{\vec{j}}
$$

\section{APPENDIX B: THE NON-COMMUTATIVE 2-SPHERE}

A non-commutative space $X$ is a space which is defined by specifying the (non-commutative) algebra of square integrable functions on it $L^{2}(X)$. In this part we recall some facts about the construction of the non-commutative sphere. The sphere $S^{2}$ can be described by the algebra $L^{2}\left(S^{2}\right)$. A basis of this algebra is given by polynomes of $\left(x_{1}, x_{2}, x_{3}\right)$ such that

$$
x_{1}^{2}+x_{2}^{2}+x_{3}^{2}=1
$$

Another possible basis is given the spherical harmonics $Y^{l m}$ and arises from the decomposition

$$
L^{2}\left(S^{2}\right)=\bigoplus_{l=1}^{+\infty} V_{l} .
$$

where $V_{l}$ is the spin $j$ representation of $\mathrm{SO}(3)$. Let us construct the non-commutative sphere $S_{N}^{2}$ (for a deformation parameter $1 / N$ ) by giving its algebra of functions, obtained by starting from the first basis described for the sphere. Let us consider $V_{\frac{N}{2}}$, the spin $N / 2$ representation of the algebra $\mathrm{su}(2) ; D^{N / 2}$ are the matrices of representation. Let us define

$$
X_{i}=\frac{1}{N} D^{N / 2}\left(J_{i}\right)
$$

where the $J_{i}$ are the generators of $\mathrm{su}(2) . X_{i}$ is in $V_{\frac{N}{2}}$. Due to the Lie algebra relations of $\mathrm{su}(2)$, one gets

$$
\begin{aligned}
\sum_{i} X_{i} X^{i} & =1 \\
{\left[X_{i}, X_{j}\right] } & =\frac{1}{N} \epsilon_{i j k} X_{k}
\end{aligned}
$$

The first relation is the analogue of the relation (eq. B1), and the second one says that the $X_{i}$ are commuting in the large $N$ limit. This facts lead us to see the $X_{i}$ as non-commuting spherical coordinates, and to define the algebra of functions on the non-commutative sphere as the algebra of endomorphism of $V_{\frac{N}{2}}$

$$
L^{2}\left(S_{N}^{2}\right)=\operatorname{End}\left(V_{\frac{N}{2}}\right) \sim V_{\frac{N}{2}} \otimes V_{\frac{N}{2}}^{*}
$$

The right-hand side can be written as

$$
\bigoplus_{l=0}^{N} V_{l}
$$

which shows that the parameter $N$ is nothing more than the cut-off in the decomposition of the functions over the sphere into spherical harmonics. As the endomorphisms of $V_{\frac{N}{2}}$ are the non-commutative counterpart of the polynomials over the sphere, a natural question is to ask what are the non-commutative counterpart of the spherical harmonics basis. Indeed, each spherical harmonic $Y^{l, m}$ for $l \leq N$ can be represented as an endomorphism of $V_{\frac{N}{2}}$ by

$$
Y^{l, m} \longrightarrow\left[\hat{Y}^{l m}\right]_{i j}=C_{m}^{l}{ }_{i}^{\frac{N}{2}}{ }^{\frac{N}{2}} .
$$




\section{APPENDIX C: NOTE ON THE CONVERGENCE OF GRAPHS}

If we consider the amplitude associated to a graph $\Gamma$ in the group field theory obtained by using $p$ times the projection (see subsection II C), we get

$$
\mathcal{A}[\Gamma]=\sum_{\{j\}} \prod_{f} d_{j_{f}} \prod_{e} \Theta^{p}\left(j_{1}, j_{2}, j_{3}\right) \prod_{v}\left\{\begin{array}{lll}
j_{1} & j_{2} & j_{3} \\
j_{4} & j_{5} & j_{6}
\end{array}\right\}
$$

We have proven for the $\Theta$ the following bound

$$
\Theta\left(j_{1}, j_{2}, j_{3}\right) \leq \frac{1}{\left(d_{j_{1}} d_{j_{2}} d_{j_{3}}\right)^{1 / 3}} .
$$

We consider also the bound on the $6 j$-symbol

$$
\left|\left\{\begin{array}{lll}
j_{1} & j_{2} & j_{3} \\
j_{4} & j_{5} & j_{6}
\end{array}\right\}\right| \leq \frac{1}{\left(\prod_{i}^{6} d_{j_{i}}\right)^{1 / 6}}
$$

obtained from the orthogonality relation (A4).

The amplitude $(\mathrm{C} 1)$ can be bounded

$$
\mathcal{A}[\Gamma] \leq \sum_{\left\{j_{f}\right\}} \prod_{f} d_{j_{f}}^{1-\frac{p}{3} n_{e}(f)-\frac{1}{6} n_{v}(f)}
$$

where $n_{e}(f)$ and $n_{v}(f)$ are the number of edges and vertices lying in the face $f$. As these numbers are equal, we obtain the bound

$$
\mathcal{A}[\Gamma] \leq \prod_{f}\left(\sum_{j_{f}} d_{j_{f}}^{1-\frac{2 p+1}{6} n_{v}(f)}\right) .
$$

One can see that there is no divergence associated to the sum over spins of face $f$ if the number of vertices lying in this face is such that

$$
n_{v}(f)>\frac{12}{2 p+1}
$$

For instance in the case of $p=2$, we obtain that the only graphs which are not proved to be convergent are for the faces such that $n_{v}(f)=1,2$. The dual faces bounded by only one or two dual vertices correspond to edges bounded by only one or two tetrahedra in the triangulation. This observation justify that only these two types of graphs need to be renormalized. However, our bounds are not optimal and numerical simulations suggest that all the graphs actually converge for $p=2$, while for $p=1$, the graphs with $n_{f}(v)=1,2$ diverges.

[1] G. T. Horowitz, Topology change in classical and quantum gravity, Class. Quant. Grav. 8 587-602 (1991), hep-th/9109030.

[2] G. Ponzano and T. Regge, Semiclassical limit of Racah coefficients, in Spectroscopic and Group Theoretical Methods in Physics, ed. F. Bloch (North-Holland, New-York, 1968).

[3] J. C. Baez, An Introduction to Spin Foam Models of Quantum Gravity and BF Theory Lect.Notes Phys. 543 (2000) 25-94 gr-qc/9905087.

[4] D. Oriti, Spacetime geometry from algebra : spin foam models for non-perturbative quantum gravity, Rept.Prog.Phys. 64 (2001) 1489-1544, gr-qc/0106091.

[5] J. Ambjorn, B. Durhuus, T. Johnson, Three-dimensional simplicial quantum gravity and generalized matrix models, Mod. Phys. Lett. A6 (1991) 1133-1146.

[6] F. J. Dyson, Divergence of perturbation theory in quantum electrodynamics, Phys. Rev. 85 (1952) 631-632, available in [13]

[7] P. Di Francesco, P. Ginsparg, J. Zinn-Justin, 2D gravity and random matrices, Phys.Rept. 254 (1995) 1-133 hep-th/9306153.

[8] D. V. Boulatov, A model of three-dimensional lattice gravity, Mod. Phys. Lett A7 (1992) 1629-1646, hep-th/9202074.

[9] H. Ooguri, Topological lattice models in four dimensions, Mod. Phys. Lett A7 (1992) 2799-2810, hep-th/9205090.

[10] R. De Pietri, C. Petronio, Feynman diagrams of generalized matrix models and the associated manifolds in dimension 4, J. Math. Phys. 41 (2000) 6671-6688, gr-qc/0004045. 
[11] R. De Pietri, L. Freidel, K. Krasnov, C. Rovelli, Barrett-Crane model from a Boulatov-Ooguri field theory over a homogeneous space, Nucl.Phys. B574 (2000) 785-806 hep-th/9907154.

[12] M. P. Reisenberger, C. Rovelli, Spacetime as a Feynman diagram: the connection formulation, Class.Quant.Grav. 18 (2001) 121-140, gr-qc/0002095.

[13] J. C. Le Guillou and J. Zinn-Justin, Eds, Large-order behaviour of perturbation theory, Current physics sources and comments Vol. 7 (North-Holland, Amsterdam, 1990).

[14] A. Perez, C. Rovelli, A spin foam model without bubble divergences, Nucl.Phys. B599 (2001) 255-282, gr-qc/0006107.

[15] A. Perez, Finiteness of a spinfoam model for euclidean quantum general relativity, Nucl.Phys. B599 (2001) 427-434 gr-qc/0011058.

[16] S. Ramgoolam, On spherical harmonics for fuzzy spheres in diverse dimensions, Nucl.Phys. B610 (2001) 461-488, hepth/0105006.

[17] J. Madore, The fuzzy sphere, Class. Quant. Grav. 9 (1992) 69-87.

[18] L. Freidel, K. Krasnov, Simple Spin Networks as Feynman Graphs, J.Math.Phys. 41 (2000) 1681-1690, hep-th/9903192.

[19] D. Oriti, Boundary terms in the Barrett-Crane spin foam model and consistent gluing, Phys.Lett. B532 (2002) 363-372, gr-qc/0201077.

[20] A. D. Sokal, An improvement of Watson's theorem on Borel summability, J. Math. Phys. 21 (1980) $261-263$. 